\title{
Diagnostic Accuracy of 18F-FDG-PET/CT and MRI in Predicting the Tumor Response in Locally Advanced Cervical Carcinoma Treated by Chemoradiotherapy: A Meta-Analysis
}

\author{
Sharareh Sanei Sistani $\mathbb{( D},{ }^{1}$ Fateme Parooie $\mathbb{D}^{1},{ }^{2}$ and Morteza Salarzaei $\mathbb{D}^{2}$ \\ ${ }^{1}$ Department of Radiology, School Medicine, Zahedan University of Medical Science, Zahedan, Iran \\ ${ }^{2}$ Student Research Committee, Zabol University of Medical Science, Zabol, Iran \\ Correspondence should be addressed to Morteza Salarzaei; mr.mortezasalar@gmail.com
}

Received 11 September 2020; Revised 17 January 2021; Accepted 20 February 2021; Published 2 March 2021

Academic Editor: Changning Wang

Copyright ( $) 2021$ Sharareh Sanei Sistani et al. This is an open access article distributed under the Creative Commons Attribution License, which permits unrestricted use, distribution, and reproduction in any medium, provided the original work is properly cited.

\begin{abstract}
Objective. The aim of this meta-analysis was to compare the diagnostic accuracy of 18F-FDG-PET/CT and MRI in predicting the tumor response in locally advanced cervical carcinoma (LACC) treated by chemoradiotherapy (CRT). Method. This meta-analysis has been performed according to PRISMA guidelines. Systematic searches were conducted using PubMed and Embase databases for articles published from January 1, 2010, to January 1, 2020. By using the Quality Assessment of Diagnostic Accuracy Studies 2 (QUADAS-2) tool, the reviewers assessed the methodological quality scores of the selected studies. We analyzed the sensitivity, specificity, and accuracy of two diagnostic methods using Meta-DiSc 1.4 and Stata 15. Results. An overall of 15 studies including 1132 patients were included. Sensitivities of PET/CT and MRI were $83.5 \%$ and $82.7 \%$, while the corresponding rates for specificities were $77.8 \%$ and $68.4 \%$, respectively. The DOR, PLR, and NLR for MRI were 15.140, 2.92, and 22.6. PET/CT had a DOR of 25.21. The PLR and NLR for PET/CT were 4.13 and 0.215 , respectively. The diagnostic sensitivity and specificity of PET/CT for the detection of residual tumor were $86 \%$ and $95 \%$, respectively. The corresponding rates for MRI were $73 \%$ and $96 \%$, respectively. The diagnostic sensitivity and specificity of PET/CT for the detection of tumor metastases were $97 \%$ and $99 \%$, while the corresponding rates for MRI were $31 \%$ and $98 \%$, respectively. Conclusion. 18 F-FDG PET/CT seemed to have a better overall diagnostic accuracy in the evaluation of treatment response to chemoradiotherapy in LACC patients. MRI showed a really poor sensitivity in the detection of metastases, and PET/CT performed significantly better. However, the difference between these two methods in the detection of residual disease was not significant. More studies are needed to be conducted in order to approve that 18F-FDG PET/CT can be a standard option to assess the treatment response.
\end{abstract}

\section{Introduction}

Cervical cancer is one of the most prevalent cancers among women [1]. About $40 \%$ of these patients show locally advanced cervical carcinoma (LACC) at initial diagnosis [2]. The standard treatment for LACC is specific cisplatin-based radiotherapy chemotherapy (CRT) [3]. However, usually, in $33 \%$ of cases, the tumor recurs about 2 years after CRT, and the overall 5-year survival is approximately $70 \%$ [3]. Lymph node status, response to treatment, and clinical stage are the main predictors of recurrence. Among the new strategies, neoadjuvant CRT followed by radical surgery is performed with the purpose of removing residual tumors which are potentially radio- and chemo-resistant and also improving local control and survival $[4,5]$. In addition, radical surgery provides useful prognostic information, which is the pathological response to treatment. Women who obtain a complete pathological response to neoadjuvant CRT showed significantly longer overall survival and disease-free survival than women with partial response [6,7]. However, applying this approach has some intraoperative and postoperative complications [8]. Therefore, there is a need for imaging techniques that can evaluate the tumor response accurately during and after treatment, so personalized treatment can be 
made possible. Imaging techniques such as magnetic resonance imaging (MRI), computed tomography (CT), and positron emission tomography (PET) with 18F-fluorodeoxyglucose (FDG) are more useful tools for evaluating how extensive the tumor is [9]. In the evaluation of tumor size and invasion and the local extent of the disease, MRI is superior to CT. Unlike CT and MRI, PET is used to evaluate tumor metabolic function. Pathological tumor size in cervical cancer and tumor size measured by PET have been shown to have strong correlation [10]. PET has been used to evaluate pretreatment situation and daily surveillance of patients with cervical cancer after treatment [11]. 3-month posttreatment PET can be applied to predict the therapeutic response [12]. However, patients who have a poor response to CRT can be detected as soon as possible, and their treatment plan can be changed as well, provided that the tumor response can be estimated during or just after concomitant chemoradiotherapy. 18F-FDG-PET/CT is important in LACC staging due to its capability to detect distant metastases and involved lymph nodes; it, therefore, improves treatment planning [13]. Furthermore, 18F-FDG-PET/CT can predict better prognosis in patients who developed complete metabolic response when performed after exclusive CRT [13]. In contrast, the role of $18 \mathrm{~F}-\mathrm{FDG}-\mathrm{PET} / \mathrm{CT}$ performed during treatment is not defined apparently [4]. The aim of this metaanalysis is to compare the diagnostic accuracy of 18F-FDG$\mathrm{PET} / \mathrm{CT}$ and MRI in predicting the tumor response in locally advanced cervical carcinoma treated by chemoradiotherapy.

\section{Materials and Methods}

2.1. Literature Search. This meta-analysis has been performed according to Preferred Reporting Items for Systematic Reviews and Meta-Analyses guidelines [14]. Systematic searches were conducted using PubMed and Embase databases for articles published from January 1, 2010 , to January 1,2020 . The search query was done using the key terms "cervical cancer," "magnetic resonance imaging or MRI," "18F-FDG PET/CT," "accuracy," "specificity," "sensitivity," and "prognosis," and related terms are as follows: (cervix or cervical) and (PET or positron emission tomography) or (FDG or fluorodeoxyglucose). There was no limitation regarding the language of the studies. The bibliography of retrieved early studies was cross-examined in order to find other related papers and articles.

The inclusion criteria consisted of the following: studies evaluating PET/CT and MRI diagnostic accuracy in the treatment of LACC. Pathological results were considered as the "gold standard." The diagnostic criteria were as follows: there are $2 \times 2$ contingency tables (Table 1 ); direct or indirect access to true positive, false positive, true negative, false negative, specificity, and sensitivity. We also included some additional studies as they provided data which helped to complete our manuscript and make it more understandable. These data were analyzed separately from the diagnostic accuracy data. The exclusion criteria were as follows: publication type other than the authentic research papers (i.e., review articles and conference abstracts), not in the field of the researchers' interest. The searching process and selection
TABLE 1: The required data for construction of a $2 \times 2$ table.

\begin{tabular}{lccccc}
\hline Author & TP & TN & FP & FN & Imaging method \\
\hline Choi et al. [15] & 63 & 64 & 5 & 4 & PET/CT \\
Rufini et al. [16] & 30 & 29 & 11 & 18 & PET/CT \\
Cetina et al. [17] & 12 & 2 & 2 & 0 & PET/CT \\
Perrone et al. [18] & 20 & 10 & 10 & 0 & PET/CT \\
Vandecasteele et al. [19] & 16 & 18 & 4 & 2 & MRI \\
Oh et al. [20] & 8 & 40 & 9 & 1 & PET/CT \\
Shen et al. [21] & 15 & 65 & 21 & 6 & PET/CT \\
Mongula et al. [22] & 15 & 18 & 6 & 3 & MRI \\
Scarsbrook et al. [23] & 45 & 30 & 6 & 3 & PET/CT \\
Su et al. [24] & 16 & 27 & 0 & 6 & PET/CT \\
Gui et al. [25] & 7 & 27 & 0 & 20 & MRI \\
Atstupenaite et al. [26] & 2 & 27 & 11 & 1 & MRI \\
\hline
\end{tabular}

of the articles were done by two independent reviewers with 3 years of meta-analysis experience. Disagreements were resolved through discussion.

2.2. Data Extraction and Quality Assessment. The two abovementioned authors separately assessed each study and then extracted the data by applying standardized dataabstraction forms. The following characteristics were extracted: study characteristics included institution, publication year, first author, country, patient enrollment period, design (prospective or retrospective), number of patients, and reference standard, and clinical and radiologic characteristics included patient FIGO stages, histology of the tumor, age, lymph node metastasis, tumor size, treatment, 3-year overall survival (OS), disease-free interval (DFI), true positive, false positive, true negative, false negative, specificity, and sensitivity. $2 \times 2$ contingency tables were constructed, and we calculated the specificity, sensitivity, and likelihood ratios (LR). By using the Quality Assessment of Diagnostic Accuracy Studies 2 (QUADAS-2) tool, the reviewers assessed the methodological quality scores of the selected studies including 11 standard items, applying review manager software program (RevMan, version 5.0.2, Nordic Cochrane Centre, Copenhagen, Denmark). The answers "yes," "no," or "unclear" to the 11 standard questions represented that the risk of bias can be judged to be low, the potential for bias exists, or inadequate data are reported to permit a judgment, respectively.

2.3. Statistical Analysis. We analyzed the sensitivity, specificity, and accuracy of two diagnostic methods by Meta-DiSc 1.4 and Stata 15 with their $95 \%$ confidence intervals (CI). Also, we have drawn the hierarchical summary receiver operating characteristic (HSROC) curves. The random effect model or fixed effect model was used to evaluate the effect values based on the results of the heterogenicity test.

\section{Results}

After a comprehensive computerized search, searching for articles and selecting them were done, and reference lists were cross-checked, as well. We recorded 1,435 files, of 
which 347 duplicate abstracts were removed. On the other hand, 641 unrelated studies, 89 conference abstracts, 73 case reports, 16 editorials, 27 letters, and 217 review articles were excluded. The remaining 25 full-text articles were examined for eligibility, and 7 articles were excluded (lack of required data to calculate the sensitivity and specificity of $18 \mathrm{~F}-\mathrm{FDG}$ $\mathrm{PET} / \mathrm{CT}$ and MRI) to predict tumor response in LACC after CRT. Finally, 15 studies were selected to be included, and in the screening process of the references of these articles, no other eligible studies were found [20-37]. The features of the included studies are presented in Table 2. The detailed method of study selection in the current meta-analysis is shown in Figure 1.

\subsection{Literature Review}

3.1.1. PET/CT. Rufini et al., Oh et al., and Choi et al., in their studies, focused on the correlation between the results of $\mathrm{PET} / \mathrm{CT}$ and pathologic complete response. They suggested following the changes through the treatment process using delta SUV and delta TLG in order to achieve the most accurate diagnosis $[14,15,20]$. Choi et al. also suggested SUV $_{\text {max }}$ of 4.0 as an optimal cutoff on the posttreatment PET/CT [15]. Scarsbrook et al. defined a five-point qualitative response assessment scoring system using which they reached a high sensitivity for PET/CT (28).

3.1.2. MRI. Gui et al. reported a relatively low sensitivity and specificity for MRI. Their results indicated that MRI performance is not sufficient in distinguishing post-CRT inflammation from the residual tumor which can lead to a high number of false positives. However, the negative predictive value of MRI was high with a low risk of false negative [25]. The results of Atstupenaite were inline with this study and indicated high specificity and low sensitivity for MRI in postCRP evaluation of patients [26].

3.1.3. PET/CT and MRI. Perron et al. evaluated PET/CT and MRI accuracies in the same population. They reported a significantly higher Cohen Kappa coefficient between follow-up findings and PET/CT results compared to the findings of MRI. However, Vandecasteele et al., in their study on 27 cervical cancer patients, indicated a very low sensitivity for PET/CT and considered MRI as the preferred modality for recurrence assessment as it provided a specificity of $100 \%$ associated with a $74 \%$ NPV in their study $[20,21]$. However, this difference could be caused by the small sample size in Vandecasteele et al.'s study. Su et al., on the other hand, confirmed the superiority of PET/CT in post-CRP patients $(P=0.025)$ [24] (Table 1).

3.2. Study Description, Quality, and Publication Bias. All data analyses were performed on per-patient data. Out of 15 studies, 2 studies were done prospectively and 13 studies were retrospective analysis. A total of 1308 patients aged between 22 and 90 years were included in this study. The overall prevalence of patients with FIGO IB stage is $4.2 \%$ (95\% CI:
2.4-6, $\left.I^{2}: 90.3 \%\right)$ and FIGO IIA-IIB and IIIA-IIIB stages are 71.5\% (95\% CI: 67.4-75.6, $I^{2}: 85.12$ ) and 14.7\% (95\% CI: 4/ 11-18), respectively. The staging data were only provided in 5 articles including 486 patients $(17-19,22,23)$. The histopathology of $87.1 \%$ (95\% CI: 87.1-84.4, $I^{2}: 46.7 \%$ ) of tumors was reported to be squamous cell carcinoma, and $12.6 \%$ (95\% CI: 6.6-15.7, $\left.I^{2}: 55.8 \%\right)$ tumors were adenocarcinomas. The histopathologic data were only provided in 6 articles including 526 patients $(17-20,22,23)$. The main characteristics of 16 studies in a meta-analysis are given in Tables 2 and 3 .

3.3. Methodological Quality Assessment. Figure 2 indicates that the risk of bias applicability concerns summary of included studies, and in general, the quality of studies included is considered satisfactory.

\subsection{Diagnostic Accuracy of PET/CTand MRI for Predicting the} Tumor Response. Overall diagnostic sensitivities of PET/CT and MRI for predicting the tumor response in locally advanced cervical carcinoma treated by chemoradiotherapy were $82.7 \%$ (95\% CI: 75-88.6, $I^{2}: 71.9 \%$ ) and $83.5 \%$ (95\% CI: 79.9-86.6, $I^{2}: 86.7 \%$ ), and the corresponding rates for specificities were $68.4 \%$ (95\% CI: 62-74.4.1, $\left.I^{2}: 70.7 \%\right)$ and $77.8 \%$ (95\% CI: $74.4-81.1, I^{2}: 91.2 \%$ ), respectively. The DOR, PLR, and NLR for MRI were 15.140\% (95\% CI: 5.507-41.62, $\left.I^{2}: 56.7 \%\right), 2.92$ (95\% CI: 1.980-4.305, $I^{2}: 65.5 \%$ ), and 22.6 (95\% CI: $10.6-48.2, I^{2}: 64.3 \%$ ). PET/CT had a DOR of 25.21 (95\% CI: 8.27-76.88, $I^{2}: 82.1 \%$ ). The PLR and NLR for PET/ CT were 4.13 (95\% CI: 2.16-7.89, $\left.I^{2}: 91.2 \%\right)$ and 0.215 (95\% CI: $0.116-0.398, I^{2}: 86.3 \%$ ), respectively (Tables 3 and 4 , Figures 3-5). These ratios were obtained from 15 studies which consisted of 1132 patients.

3.5. Diagnostic Accuracy of PET/CTand MRI for the Detection of Residual Tumor. The diagnostic sensitivity and specificity of PET/CT for the detection of residual tumor in patients with locally advanced cervical carcinoma were $86 \%$ (95\% CI: $83-0.90, I^{2}: 92.8 \%$ ) and $95.5 \%$ (95\% CI: $93.4-98, I^{2}: 96.3 \%$ ), and the corresponding rates for MRI were 73.5\% (95\% CI: 69.3-78, $I^{2}: 87 \%$ ) and $96.2 \%$ (95\% CI: 94-98, $\left.I^{2}: 94.6 \%\right)$, respectively. These data were according to $3[26,28,29]$ and $4[26,29-31]$ articles including 293 and 290 patients for PET/CT and MRI.

3.6. Diagnostic Accuracy of PET/CTand MRI for the Detection of Tumor Metastases. The diagnostic sensitivity and specificity of PET/CT for the detection of tumor metastases in patients with locally advanced cervical carcinoma were $97 \%$ (95\% CI: 94-99, $I^{2}:$ 97.3\%) and 99\% (95\% CI: 96-100, $I^{2}$ : $00.0)$, and the corresponding rates for MRI were $31 \%(95 \%$ CI: $\left.23-39 I^{2}: 97.7 \%\right)$ and $98 \%$ (95\% CI: $\left.96-100 I^{2}: 65.3 \%\right)$ based on 2 articles including 95 patients [20,29].

3.7. Prevalence of Residual Disease, Pelvic Lymph Node Metastases, and Distant Metastases. The results of our metaanalysis revealed a prevalence of $12.7 \%$ (95\% CI: 9.3-16, $I^{2}$ : $35.9 \%$ ) for residual disease based on 4 articles including 378 
TABLE 2: Study characteristics.

\begin{tabular}{|c|c|c|c|c|c|c|}
\hline Author & Year & Duration & Country & $N$ & Mean age & Study design \\
\hline Choi et al. [15] & 2013 & $2005-2012$ & Korea & 136 & 57.1 & Retro \\
\hline Rufini et al. [16] & 2019 & 2010-2014 & Italy & 88 & $49.5(22-75)$ & Pros \\
\hline Cetina et al. [17] & 2011 & $2007-2008$ & Mexico & 60 & 47.2 & Retro \\
\hline Perrone et al. [18] & 2020 & $2007-2017$ & Italy & 40 & 55 & Retro \\
\hline Vandecasteele et al. [19] & 2012 & $2007-2010$ & Belgium & 27 & 49 & Retro \\
\hline Chen et al. [38] & 2018 & $2009-2015$ & Taiwan & 142 & 55 & Retro \\
\hline Oh et al. [20] & 2013 & 2009-2010 & Korea & 60 & 53.5 & Retro \\
\hline Lucia et al. [27] & 2017 & $2010-2016$ & France & 102 & 58 & Retro \\
\hline Miccò et al. [28] & 2014 & 2009-2012 & USA & 49 & 45 & Retro \\
\hline Shen et al. [21] & 2019 & 2019-2015 & Taiwan & 142 & 55 & Retro \\
\hline Mongula et al. [22] & 2016 & - & - & 42 & 53 & Rero \\
\hline Scarsbrook et al. [23] & 2017 & 2011-2014 & UK & 96 & 47 & Retro \\
\hline Su et al. [24] & 2018 & - & Taiwan & 55 & - & Retro \\
\hline Gui et al. [25] & 2015 & $2005-2008$ & Italy & 41 & 48 & Pro \\
\hline Atstupenaite et al. [26] & 2017 & $2009-2010$ & Lithuania & 52 & - & Retro \\
\hline
\end{tabular}

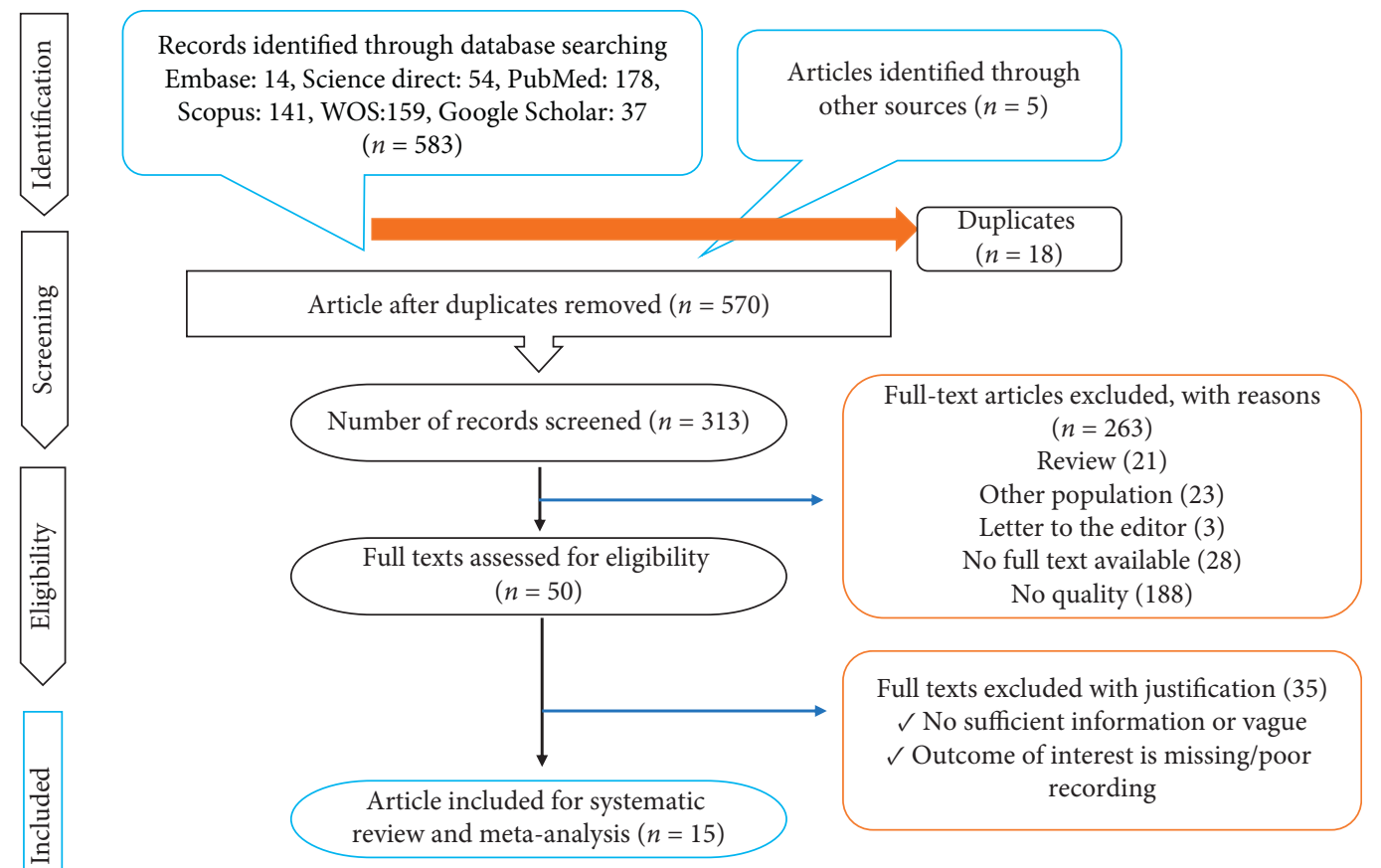

Figure 1: PRISMA flow diagram.

patients $[17,20,22,23]$, a prevalence of $49 \%$ (95\% CI: $\left.43.7-54.3, I^{2}: 81.3 \%\right)$ for pelvic lymph node metastases based on 4 articles including 330 patients [18, 20, 22, 23], and a prevalence of $11 \%$ (95\% CI: 7.9-14.1, $I^{2}: 83.8 \%$ ) for distant metastases in patients with locally advanced cervical cancer after chemo-radiotherapy based on 5 articles including 379 patients $[17,18,20,22,23]$ (Table 3).

3.8. Meta-Analysis of 3-Year Overall Survival, PFS, and Mean DFI. The overall 3-year survival, PFS, and mean DFI in patients with locally advanced cervical cancer after chemoradiotherapy were $92.4 \%$ (95\% CI: 87.3-97.6, $I^{2}$ : 0.8\%) and $74.6 \%$ (95\% CI: $\left.66.1-83.1, I^{2}: 0.0\right)$, respectively. The mean DFI for these patients was 15 months. Each of these ratios was obtained from 2 studies involving 100, 100, and 150 patients, respectively [18-23] (Table 3).

3.9. Meta-Analysis of SUV mean in Patients with Complete and Partial Response. The overall $\mathrm{SUV}_{\text {mean }}$ for predicting treatment response in patients with locally advanced cervical cancer was reported to be 1.9 for patients who developed complete response and 5.10 for patients who did not, based on 4 articles including 372 patients $[17-19,22]$ (Table 3 ).

3.10. Fagan's Nomogram for the Calculation of Posttest Probabilities. A pretest probability of $50 \%$ for all three diagnostic tools was fixed, which was estimated by the number 


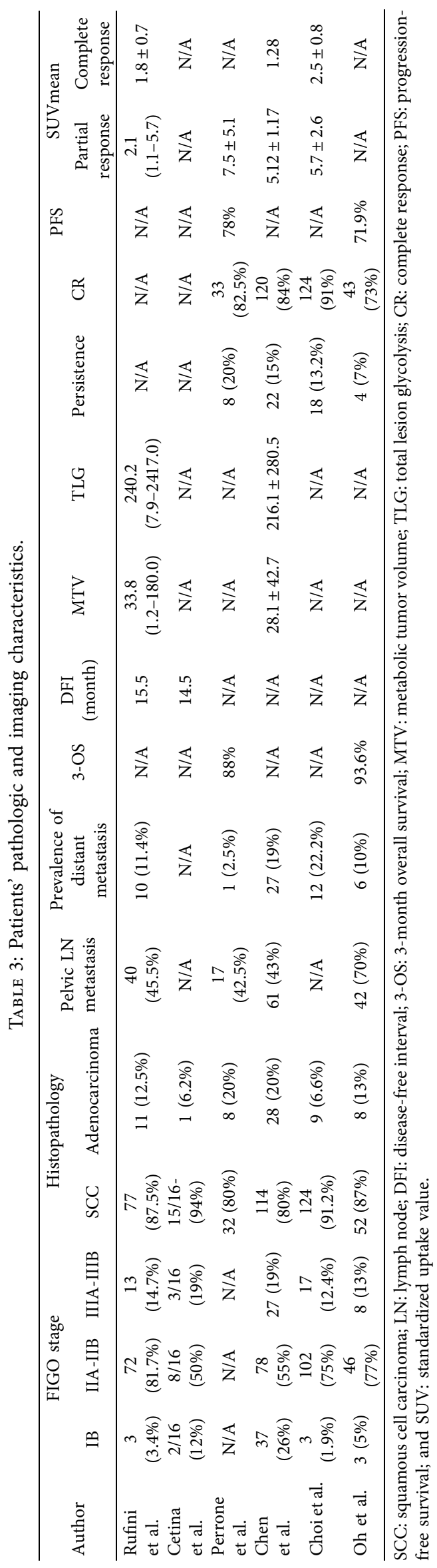




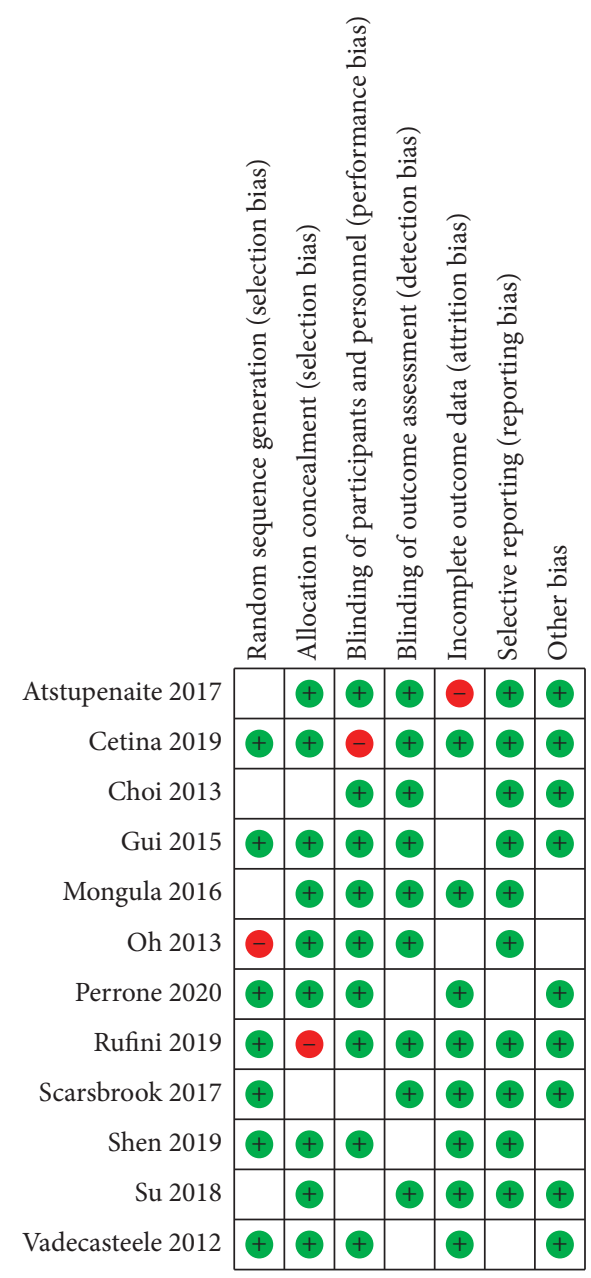

FIgURE 2: QUADS-2 for the evaluation of methodological quality.

TABle 4: Diagnostic performance of MRI and PET/CT in patients with LACC post-CRT.

\begin{tabular}{lcc}
\hline Parameter & PET/CT & MRI \\
\hline Sensitivity (95\% CI) & 0.835 (CI 95\% 0.799-0.866) & 0.827 (CI 95\% 0.75-0.88) \\
Specificity (95\% CI) & 0.778 (CI 95\% 0.744-0.811) & $0.684($ CI 95\% 0.620-0.744) \\
PLR (95\% CI) & 4.135 (CI 95\% 2.164-7.899) & $2.920($ CI 95\% $1.980-4.305)$ \\
NLR (95\% CI) & 0.215 (CI 95\% 0.116-0.398) & 0.226 CI 95\% 0.106-0.482) \\
DOR (95\% CI) & 25.216 (CI 95\% 8.270-76.887) & $15.140($ CI 95\% 5.507-41.626) \\
PPV & $75 \%$ & $85 \%$ \\
NPV & $62 \%$ & $86 \%$ \\
Accuracy & $80 \%$ & $74 \%$ \\
AUC-SROC & 0.8007 & 0.9081 \\
$I^{2}$ & $65 \%$ & $82 \%$ \\
Q index & 0.7384 & 0.8379 \\
\hline
\end{tabular}

of patients in selected studies. In Figures 4 and 6, MRI had a posttest probability of $74.1 \%$ (a). For PET/CT (b), the posttest probability was $82.4 \%$. If this patient tests positive, the posttest probability that she truly has developed residual disease or metastases would be $13.2 \%$ for MRI (a) and 99.4 for PET/CT (b) (solid line in red). The results were obtained by the following calculations: pretest odds=prevalence/ $1-$ prevalence; posttest odds $=$ pretest odds $\times \mathrm{LR}-(\mathrm{LR}+)$; posttest probability $=$ posttest odds $/ 1+$ posttest odds (Figures 4 and 6).

\section{Discussion}

Among the most common cancers in women, cervical cancer is the third malignant tumor worldwide after breast and colorectal cancer. Cervical cancer has a significant impact on women's health because of its younger onset age, high prevalence, and posttreatment recurrence. The prevalence of residual disease and lymph node metastasis after chemotherapy were $12.7 \%$ and $49 \%$, respectively. Predictors of recurrence of cervical cancer include the posttreatment 


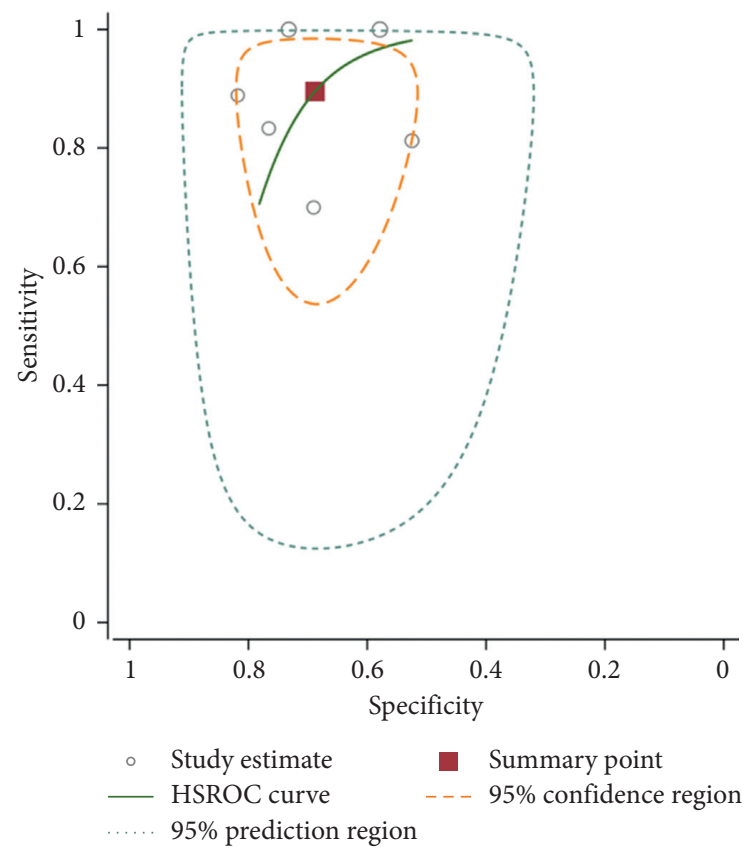

(a)

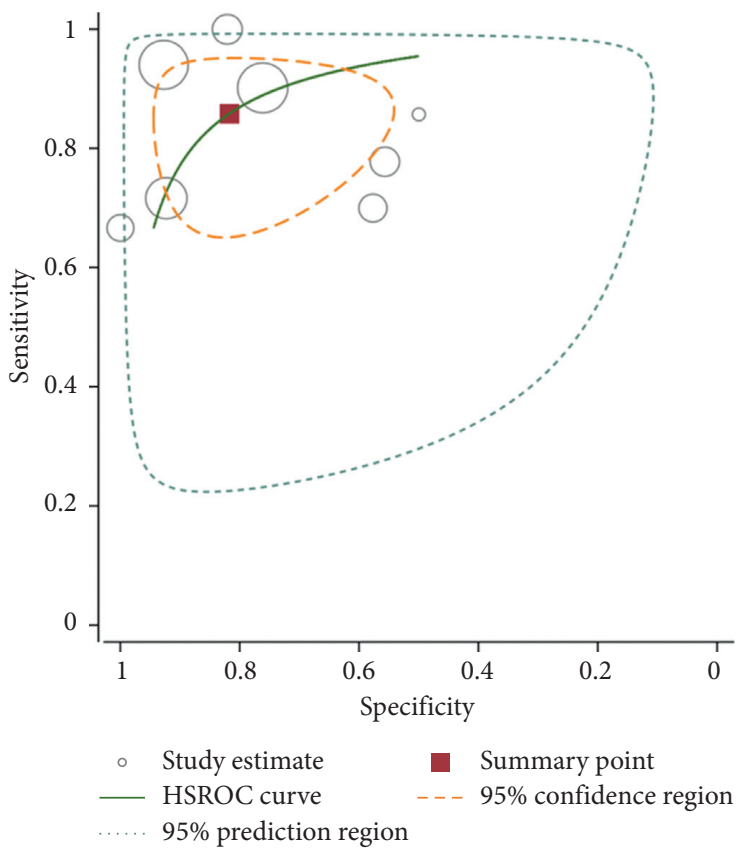

(b)

FIGURE 3: Hierarchical summary receiver (HSROC) curve for MRI (a) and 18F-FDG PET/CT (b) for predicting treatment response in LACC patients after CRT.
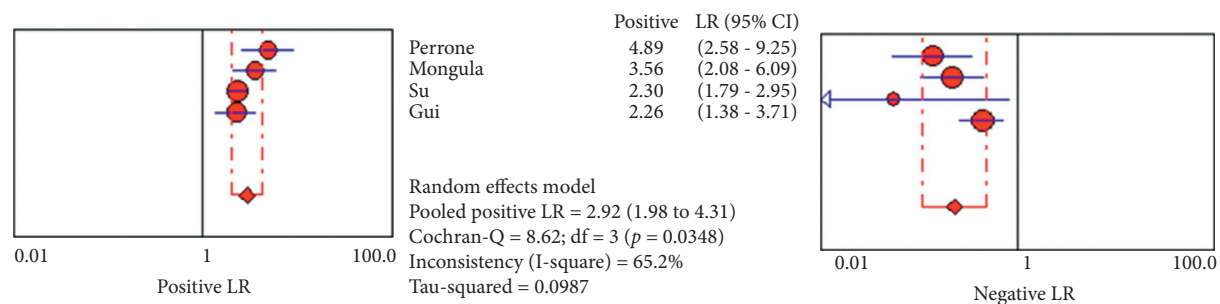

Perrone Mongula

$$
\mathrm{Su}
$$

Gui

Random effects model

Pooled negative $L R=0.23$ ( 0.11 to 0.48 )

Cochran- $\mathrm{Q}=8.41 ; \mathrm{df}=3(p=0.0383)$

Tau-squared $=0.3434$ - $=64.3 \%$

(a)

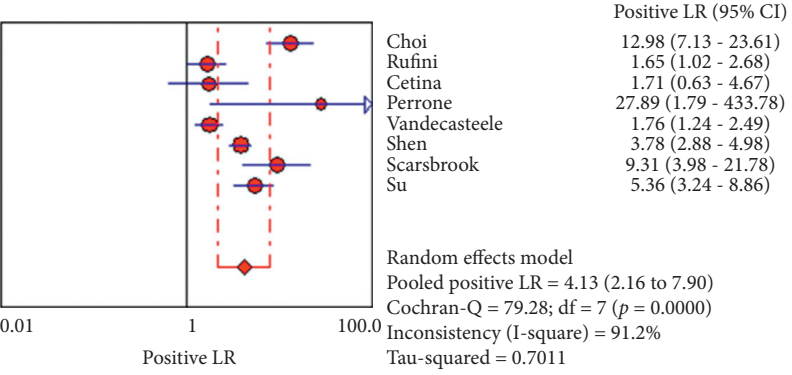

Negative LR (95\% CI)

$0.06(0.03-0.13)$ $0.52(0.20-1.37)$ $0.29(0.06-1.44)$ $0.34(0.24-0.49)$ $0.40(0.16-0.97)$ $0.13(0.07-0.23)$ $0.31(0.23-0.41)$ $0.02(0.00-0.28)$ Su

Random effects mode Pooled negative LR $=0.21(0.12$ to 0.40$)$ Cochran $-\mathrm{Q}=51.00 ; \mathrm{df}=7 \quad(p=0.0000)$

Inconsistency (I-square) $=86.3 \%$ Tau-squared $=0.5694$

(b)

FIGURE 4: PLR and NLR of 18F-FDG PET/CT (b) and MRI (a) for predicting treatment response in LACC patients after CRT based on countries.

tumor response as well as the status of the lymph nodes and clinical stage when it is diagnosed, initially [29-31]. Patients with no significant tumors after treatment are reported to have a 5 -year survival of $76 \%$. However, it has been reported that this rate is lower in patients who have findings which are suggestive of a tumor or who are diagnosed with persistent tumor (42\% and $8 \%$, respectively) [32]. According to the results of the present study, the overall 3-year survival for patients undergoing CRT was $92.4 \%$. Due to the lack of data in the studies, we could not perform a subgroup analysis to divide patients into two groups with a complete response and partial response. However, we evaluated the overall DFI and PFS of these patients, which showed that the overall DFI was $74 \%$ and the mean PFS was evaluated to be 15 months. In cervical cancer, the surgeon's goal is to rule out the progression of the disease rather than macroscopically 


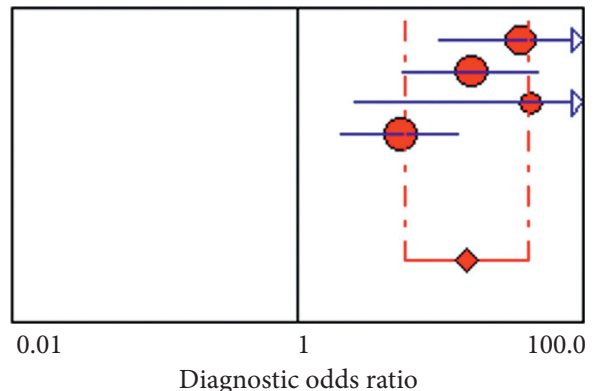

Diagnostic odds ratio

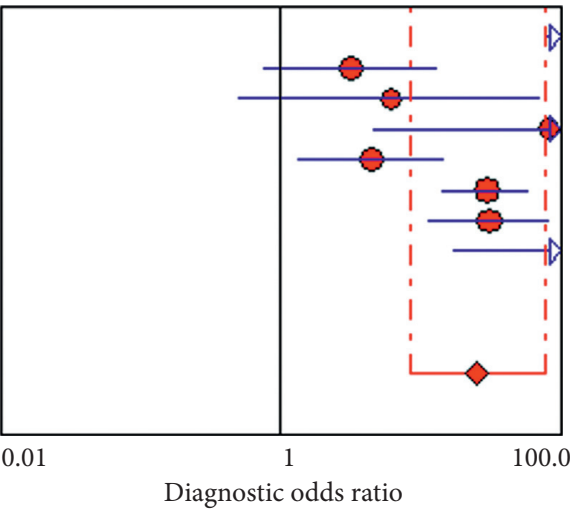

Diagnostic OR (95\% CI)

Perrone

$36.00(9.90-130.94)$
$16.36(5.41-49.48)$

$42.48(2.47-730.86)$

$5.21(2.03-13.34)$

Random effects model

Pooled diagnostic odds ratio $=15.14$ (5.51 to 41.63)

Cochran- $\mathrm{Q}=6.92 ; \mathrm{df}=3(p=0.0743)$

Inconsistency (I-square) $=56.7 \%$

Tau-squared $=0.5671$

(a)

Choi

Rufini

Cetina

Perrone

Vandecasteele

Shen

Scarsbrook

$\mathrm{Su}$

Diagnostic OR (95\% CI)

$201.60(77.06-527.40)$

$3.18(0.77-13.23)$

$6.00(0.51-70.67)$

$81.00(4.66-1,407.72)$

$4.40(1.33-14.56)$

$29.34(14.36-59.93)$

$30.33(11.26-81.68)$

297.48 (17.05 - 5,189.53)

Random effects model

Pooled diagnostic odds ratio $=25.22$ (8.27 to 76.89)

Cochran-Q = 39.06; $\mathrm{df}=7(p=0.0000)$

Inconsistency (I-square) $=82.1 \%$

Tau-squared $=1.8745$

(b)

FIGURE 5: DOR of 18F-FDG PET/CT (b) and MRI (a) for predicting treatment response in LACC patients after CRT based on countries.

invasive because this treatment is not selective on parameters, but is provided by surgery. In cervical cancer, the role of pretreatment 18F-FDG-PET/CT and MRI is well defined. In case of suspected involvement in bladder, cervix, vagina, or rectum, MRI performs better $[33,34]$. However, in the diagnosis of lymph node metastases or mesenteric, peritoneal, gastrointestinal, mediastinal, and pleural involvement, 18F-FDG-PET/CT is a more sensitive diagnostic tool [35]. Therefore, both of these diagnostic tools seem to be useful in choosing the treatment and in radiation therapy planning $[36,37]$. However, studies assessing the response to chemotherapy are few, although this evaluation plays an important role in deciding on subsequent treatments $[18,39,40]$. Clinical and radiological features are required to diagnose residual disease. Unfortunately, it is difficult to perform gynecological examinations after radiation therapy. In fact, the accurate visualization of the cervix is interfered by vaginal adhesions and fibrosis after radiation [41]. MRI after radiotherapy may not assess the response, accurately due to heterogeneous gad-contrast enhancement and inflammation in areas which are hyperintense in T2W [42]. The evaluation of 18F-FDG-PET/CT can also be interfered by the inflammation and necrosis caused by radiotherapy [43]. Therefore, it is required to define the roles of these imaging modalities after chemoradiotherapy. In this metaanalysis, we assessed the role of 18F-FDG-PET/CT and MRI in predicting tumor response in LACC after CRT. This metaanalysis includes a total of 15 studies. Due to the fact that most individual studies have a limited number of cases, more data can be used in meta-analysis and also more reliable results are provided. Our study indicated better diagnostic sensitivity for MRI follow-up data (0.86) compared to PET/ CT (0.83) in assessing the response to chemoradiotherapy for primary tumor and distant metastasis. However, the sensitivity of these two methods is quite similar, and there is no statistically significant difference between them. We also performed a subgroup analysis to decrease heterogeneity assessing the accuracy of these two methods among patients with residual disease compared to patients with distant metastases. MRI showed a real poor sensitivity in the detection of metastases (31\% vs. 97\%), and PET/CT performed significantly better. However, the difference among these two methods in the detection of residual disease was not significant (73\% vs. $86 \%$ ). Woo et al., in their meta-analysis, reported $73 \%$ sensitivity and $93 \%$ specificity for MRI in the diagnosis of parametric lesions in patients with cervical cancer [44], and Sakurai et al. declared that metabolic activity and standardized uptake value (SUV) depend on the tumor lesion $(>1 \mathrm{~cm})$; SUV was reported to have an average of 3.90 and 2.31 in tumoral and nontumoral lesions, respectively $(P>0.05)$ [45]. In our study, the mean SUV was 1.9 for patients who had a complete response and 10.10 for those who did not. Rufini et al. concluded that it is possible to evaluate the metabolic changes driven by treatment by performing 18F-FDG-PET/CT before, after, and during treatment in patients with LACC and assessing delta SUV 


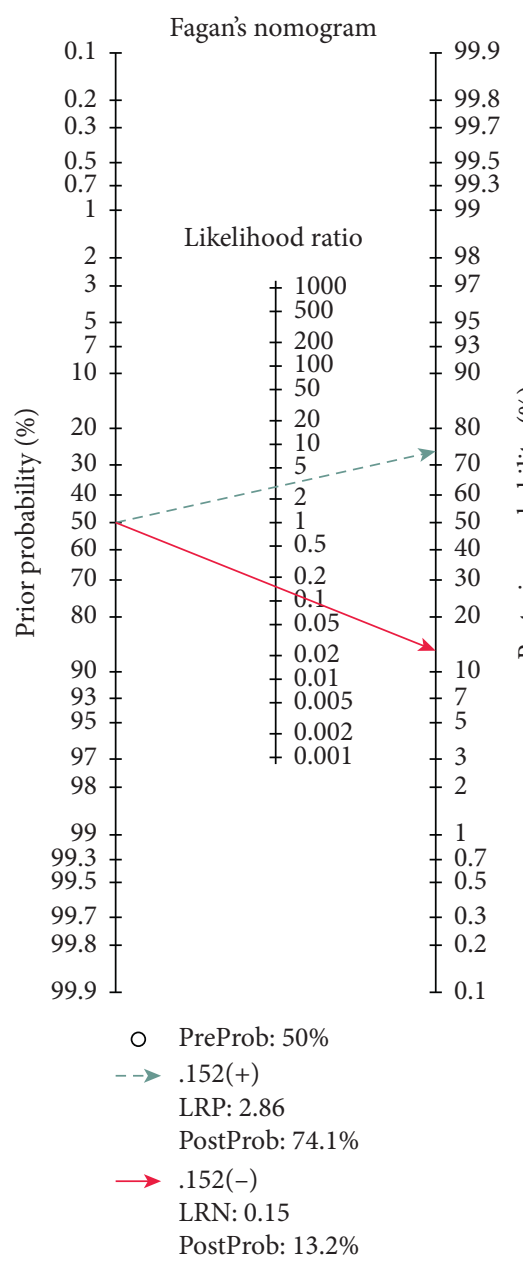

(a)

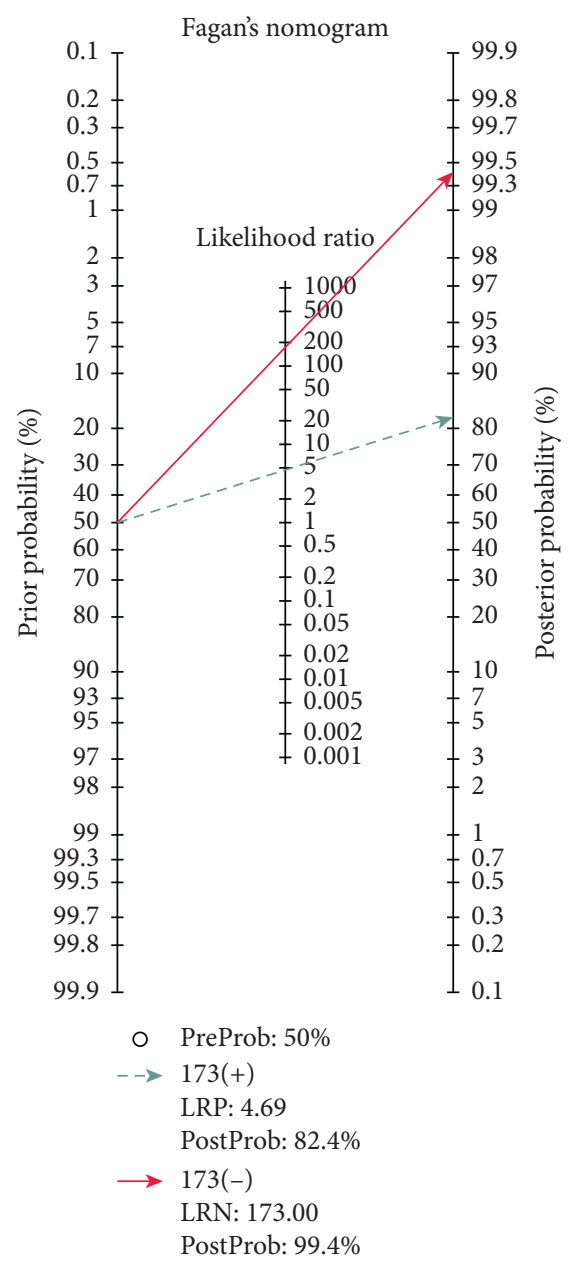

(b)

FIGURE 6: Fagan's nomogram for the calculation of posttest probabilities of MRI (a) and PET/CT (b).

parameters. However, they showed that the final assessment was not accurate enough to predict the pathological CR of the primary tumor [16]. Another study examined 25 patients with LACC (stages IB2-IIIB), evaluated them using both MRI and 18F-FDG PET/CT, before and after CRT, and indicated that $18 \mathrm{~F}-\mathrm{FDG}$ PET/CT provided important information which led to treatment planning changes in half of the patients. However, MRI detected the pelvic tumors in 2 patients which were missed by 18 F-FDG-PET/CT [42]. In contrast, a meta-analysis of 15 studies represented by Maedes et al. assessed the additional diagnostic accuracy of 18F-FDG PET/CT in the whole body compared to conventional imaging in women with suspected recurrent/ persistent cervical cancer. He reported that using 18F-FDG $\mathrm{PET} / \mathrm{CT}$ in these patients was not supported by previous studies. However, the included studies in his meta-analysis had not compared PET/CT with MRI or CT [46]. This metaanalysis had several limitations. Firstly, not all studies in this meta-analysis reported the specific techniques applied. Some scanning parameters may affect the accuracy of PET/CT and MRI. Secondly, due to the small number of published articles in this field, there is a possibility of bias in the present study. Thirdly, as there were not enough articles comparing these modalities in the same sample size, we had to include articles evaluating one of the modalities and then compare their results in a meta-analysis. This can be our major source of bias. Finally, the number of patients in the included studies was relatively small, which may lead to bias in the final results.

\section{Conclusion}

18F-FDG PET/CT seems to have a better overall diagnostic accuracy in the evaluation of the treatment response to chemotherapy in LACC patients. MRI showed a really poor sensitivity in the detection of metastases, and PET/CT performed significantly better. However, the difference between these two methods in the detection of residual disease was not significant. More studies need to be conducted in order to approve that 18F-FDG PET/CT can be a standard option to assess the treatment response.

\section{Abbreviations}

18F-FDGPET/CT:
18F-fluorodeoxyglucose positron emission tomography/computerized tomography 


$\begin{array}{ll}\text { MRI: } & \text { Magnetic resonance imaging } \\ \text { LACC: } & \text { Locally advanced cervical cancer } \\ \text { CRT: } & \text { Chemoradio therapy } \\ \text { QUADAS-2: } & \begin{array}{l}\text { Quality Assessment of Diagnostic Accuracy } \\ \text { Studies 2 }\end{array} \\ \text { DOR: } & \text { Diagnostic odds ratio } \\ \text { PLR: } & \text { Positive likelihood ratio } \\ \text { NLR: } & \text { Negative likelihood ratio } \\ \text { CT: } & \text { Computed tomography } \\ \text { PET: } & \text { Positron emission tomography } \\ \text { DFI: } & \text { Disease-free interval } \\ \text { PFS: } & \text { Progression-free survival } \\ \text { OS: } & \text { Overall survival } \\ \text { CI: } & \text { Confidence interval } \\ \text { SUV: } & \text { Standardized uptake value. }\end{array}$

\section{Data Availability}

The data used to support the findings of this study are available from the corresponding author upon request.

\section{Conflicts of Interest}

The authors declare no conflicts of interest.

\section{Authors' Contributions}

SS and FP participated in conception and design of the study, library searches and assembling relevant literature, critical review of the paper, supervising writing of the paper, and database management. MS participated in data collection, library searches and assembling relevant literature, writing the paper, and critical review of the paper. All authors have read and approved the final version of manuscript.

\section{References}

[1] G. Chen, P. Zheng, L. Gao, J. Zhao, Y. Wang, and W. Qin, "Prevalence and genotype distribution of human papillomavirus in women with cervical cancer or cervical intraepithelial neoplasia in Henan province, central China," Journal of Medical Virology, vol. 32, 2020.

[2] E. T. Donkoh, F. Agyemang-Yeboah, R. H. Asmah, and E. K. Wiredu, "Prevalence of cervical cancer and pre-cancerous lesions among unscreened Women in Kumasi, Ghana," Medicine, vol. 98, no. 13, 2019.

[3] H. Leray, E. Gabiache, F. Courbon et al., "FDG-PET/CT identifies predictors of survival in patients with locally advanced cervical carcinoma and para-aortic lymph node involvement to increase treatment," Journal of Nuclear Medicine, vol. 12, 2020.

[4] A. Stecco, F. Buemi, A. Cassarà et al., "Comparison of retrospective PET and MRI-DWI (PET/MRI-DWI) image fusion with PET/CT and MRI-DWI in detection of cervical and endometrial cancer lymph node metastases," La Radiologia Medica, vol. 121, no. 7, pp. 537-545, 2016.

[5] Z. Zhou, X. Liu, K. Hu, and F. Zhang, "The clinical value of $\mathrm{PET}$ and PET/CT in the diagnosis and management of suspected cervical cancer recurrence," Nuclear Medicine Communications, vol. 39, no. 2, pp. 97-102, 2018.
[6] L. A. Min, L. L. Ackermans, M. E. Nowee et al., "Pre-treatment prediction of early response to chemoradiotherapy by quantitative analysis of baseline staging FDG-PET/CT and MRI in locally advanced cervical cancer," Acta Radiologica, vol. 9, 2020.

[7] M. Atri, Z. Zhang, F. Dehdashti et al., "Utility of PET-CT to evaluate retroperitoneal lymph node metastasis in advanced cervical cancer: results of ACRIN6671/GOG0233 trial," Gynecologic Oncology, vol. 142, no. 3, pp. 413-419, 2016.

[8] V. R. Bollineni, S. Ytre-Hauge, A. Gulati et al., "The prognostic value of preoperative FDG-PET/CT metabolic parameters in cervical cancer patients," European Journal of Hybrid Imaging, vol. 2, no. 1, p. 24, 2018.

[9] D. F. Pinho, B. King, Y. Xi, K. Albuquerque, J. Lea, and R. M. Subramaniam, "Value of intratumoral metabolic heterogeneity and quantitative 18F-FDG PET/CT parameters in predicting prognosis for patients with cervical cancer," American Journal of Roentgenology, vol. 214, no. 4, pp. 908916, 2020.

[10] A. Lin, S. Ma, F. Dehdashti et al., "Detection of distant metastatic disease by positron emission tomography with 18F-fluorodeoxyglucose (FDG-PET) at initial staging of cervical carcinoma," International Journal of Gynecologic Cancer, vol. 29, no. 3, 2019.

[11] J. Leseur, G. Roman-Jimenez, A. Devillers et al., "Pre- and pertreatment $18 \mathrm{~F}-\mathrm{FDG}$ PET/CT parameters to predict recurrence and survival in cervical cancer," Radiotherapy and Oncology, vol. 120, no. 3, pp. 512-518, 2016.

[12] F. S. Mohammed, N. A. Chalabi, N. N. Halim, Z. M. Metwally, and A. A. Elsayed, "Role of dynamic contrast enhanced MRI and DWI in the pre-operative assessment of endometrial \& cervical carcinoma," QJM: An International Journal of Medicine, vol. 113, 2020.

[13] E. J. Diver, E. M. Hinchcliff, A. A. Gockley et al., "Utilization of preoperative PET-CT and pelvic MRI reduces multimodality therapy in the care of women with early-stage cervical carcinoma," Gynecologic Oncology, vol. 149, pp. 35-36, 2018.

[14] A. C. Tricco, E. Lillie, W. Zarin et al., "PRISMA extension for scoping reviews (PRISMA-ScR): checklist and explanation," Annals of Internal Medicine, vol. 169, no. 7, pp. 467-473, 2018.

[15] J. Choi, H. J. Kim, Y. H. Jeong et al., "The role of 18 F-FDG $\mathrm{PET} / \mathrm{CT}$ in assessing therapy response in cervix cancer after concurrent chemoradiation therapy," Nuclear Medicine and Molecular Imaging, vol. 48, no. 2, pp. 130-136, 2014.

[16] V. Rufini, A. Collarino, M. L. Calcagni et al., "The role of 18 F-FDG-PET/CT in predicting the histopathological response in locally advanced cervical carcinoma treated by chemoradiotherapy followed by radical surgery: a prospective study," European Journal of Nuclear Medicine and Molecular Imaging, vol. 1, 2019.

[17] L. Cetina, A. Serrano, D. Cantú-De-Leon et al., "F18-FDG$\mathrm{PET} / \mathrm{CT}$ in the evaluation of patients with suspected recurrent or persistent locally advanced cervical carcinoma," Revista de Investigación Clínica, vol. 63, no. 3, pp. 227-235, 2011.

[18] A. M. Perrone, G. Dondi, M. Coe et al., "Predictive role of MRI and 18F FDG PET response to concurrent chemoradiation in T2b cervical cancer on clinical outcome: a retrospective single center study," Cancers, vol. 12, no. 3, p. 659, 2020.

[19] K. Vandecasteele, L. Delrue, B. Lambert et al., "Value of magnetic resonance and 18FDG PET/CT in predicting tumor response and resectability of primary locally advanced cervical cancer after treatment with intensity-modulated arc therapy: a 
prospective pathology-matched study," International Journal of Gynecologic Cancer, vol. 22, no. 4, 2012.

[20] D. Oh, J. E. Lee, S. J. Huh et al., "Prognostic significance of tumor response as assessed by sequential 18F-fluorodeoxyglucose-positron emission tomography/computed tomography during concurrent chemoradiation therapy for cervical cancer," International Journal of Radiation Oncology ${ }^{*}$ Biology* Physics, vol. 87, no. 3, pp. 549-554, 2013.

[21] W.-C. Shen, S.-W. Chen, K.-C. Wu et al., "Prediction of local relapse and distant metastasis in patients with definitive chemoradiotherapy-treated cervical cancer by deep learning from [18F]-fluorodeoxyglucose positron emission tomography/computed tomography," European Radiology, vol. 29, no. 12, pp. 6741-6749, 2019.

[22] J. Mongula, B. Slangen, D. Lambregts et al., "Predictive criteria for MRI-based evaluation of response both during and after radiotherapy for cervical cancer," Journal of Contemporary Brachytherapy, vol. 3, no. 3, p. 181, 2016.

[23] A. Scarsbrook, S. Vaidyanathan, F. Chowdhury, S. Swift, R. Cooper, and C. Patel, "Efficacy of qualitative response assessment interpretation criteria at $18 \mathrm{~F}-\mathrm{FDG}$ PET-CT for predicting outcome in locally advanced cervical carcinoma treated with chemoradiotherapy," European Journal of Nuclear Medicine and Molecular Imaging, vol. 44, no. 4, pp. 581-588, 2017.

[24] T.-P. Su, G. Lin, Y.-T. Huang et al., "Comparison of positron emission tomography/computed tomography and magnetic resonance imaging for posttherapy evaluation in patients with advanced cervical cancer receiving definitive concurrent chemoradiotherapy," European Journal of Nuclear Medicine and Molecular Imaging, vol. 45, no. 5, pp. 727-734, 2018.

[25] B. Gui, A. L. Valentini, M. Miccò et al., "Cervical cancer response to neoadjuvant chemoradiotherapy: MRI assessment compared with surgery," Acta Radiologica, vol. 57, no. 9, pp. 1123-1131, 2016.

[26] V. Atstupénaitè, I. Kraujutienè, R. J. Nakaitè, E. Bieliūnienė, and A. Basevičius, "The value of magnetic resonance imaging in the assessment of chemoradiotherapy of cancer of cervix uteri," Radiology Update, vol. 67, 2019.

[27] F. Lucia, D. Visvikis, M.-C. Desseroit et al., "Prediction of outcome using pretreatment 18F-FDG PET/CT and MRI radiomics in locally advanced cervical cancer treated with chemoradiotherapy," European Journal of Nuclear Medicine and Molecular Imaging, vol. 45, no. 5, pp. 768-786, 2018.

[28] M. Miccò, H. A. Vargas, I. A. Burger et al., "Combined pretreatment MRI and 18F-FDG PET/CT parameters as prognostic biomarkers in patients with cervical cancer," European Journal of Radiology, vol. 83, no. 7, pp. 1169-1176, 2014.

[29] A. L. Valentini, M. Miccò, B. Gui et al., "The PRICE study: the role of conventional and diffusion-weighted magnetic resonance imaging in assessment of locally advanced cervical cancer patients administered by chemoradiation followed by radical surgery," European Radiology, vol. 28, no. 6, pp. 2425-2435, 2018.

[30] B. Gui, M. Miccò, A. L. Valentini et al., "Prospective multimodal imaging assessment of locally advanced cervical cancer patients administered by chemoradiation followed by radical surgery-the "PRICE" study 2: role of conventional and DWMRI," European Radiology, vol. 29, no. 4, pp. 2045-2057, 2019.

[31] M. Vu, J. Yu, O. A. Awolude, and L. Chuang, "Cervical cancer worldwide," Current Problems in Cancer, vol. 42, no. 5, pp. 457-465, 2018.

[32] R. Nitecki, P. T. Ramirez, M. Frumovitz et al., "Survival after minimally invasive vs open radical hysterectomy for earlystage cervical cancer: a systematic review and meta-analysis," JAMA Oncology, vol. 6, 2020.
[33] J. J. Park, C. K. Kim, and B. K. Park, "Prediction of disease progression following concurrent chemoradiotherapy for uterine cervical cancer: value of post-treatment diffusionweighted imaging," European Radiology, vol. 26, no. 9, pp. 3272-3279, 2015.

[34] S. J. Freeman, A. M. Aly, M. Y. Kataoka, H. C. Addley, C. Reinhold, and E. Sala, "The revised FIGO staging system for uterine malignancies: implications for MR imaging," Radiographics, vol. 32, no. 6, pp. 1805-1827, 2012.

[35] T.-C. Yen, K.-K. Ng, S.-Y. Ma et al., "Value of dual-phase 2Fluoro-2-Deoxy-d-Glucose positron emission tomography in cervical cancer," Journal of Clinical Oncology, vol. 21, no. 19, pp. 3651-3658, 2003.

[36] J. Kusmirek, J. Robbins, H. Allen, L. Barroilhet, B. Anderson, and E. A. Sadowski, "PET/CT and MRI in the imaging assessment of cervical cancer," Abdominal Imaging, vol. 40, no. 7, pp. 2486-2511, 2015.

[37] S. Cima, A. M. Perrone, P. Castellucci et al., "Prognostic impact of pretreatment fluorodeoxyglucose positron emission tomography/computed tomography SUVmax in patients with locally advanced cervical cancer," International Journal of Gynecologic Cancer, vol. 28, no. 3, pp. 575-580, 2018.

[38] S. W. Chen, W. C. Shen, T. C. Hsieh et al., "Textural features of cervical cancers on FDG-PET/CT associate with survival and local relapse in patients treated with definitive chemoradiotherapy," Scientific Reports, vol. 8, no. 1, p. 1, 2018.

[39] G. M. Lima, A. Matti, G. Vara et al., "Prognostic value of posttreatment $18 \mathrm{~F}-\mathrm{FDG} \mathrm{PET} / \mathrm{CT}$ and predictors of metabolic response to therapy in patients with locally advanced cervical cancer treated with concomitant chemoradiation therapy: an analysis of intensity- and volume-based PET parameters," European Journal of Nuclear Medicine and Molecular Imaging, vol. 45, no. 12, pp. 2139-2146, 2018.

[40] M. Dessole, M. Petrillo, A. Lucidi et al., "Quality of life in women after pelvic exenteration for gynecological malignancies," International Journal of Gynecological Cancer, vol. 28, no. 2, pp. 267-273, 2018.

[41] V. Chiantera, M. Rossi, P. De Iaco et al., "Survival after curative pelvic exenteration for primary or recurrent cervical cancer: a retrospective multicentric study of 167 patients," International Journal of Gynecologic Cancer, vol. 24, no. 5, pp. 916-922, 2014.

[42] A. Galuppi, A. M. Perrone, M. La Macchia et al., "Local $\alpha$-tocopherol for acute and short-term vaginal toxicity prevention in patients treated with radiotherapy for gynecologic tumors," International Journal of Gynecologic Cancer, vol. 21, no. 9, pp. 1708-1711, 2011.

[43] Y. Y. Jeong, H. K. Kang, T. W. Chung, J. J. Seo, and J. G. Park, "Uterine cervical carcinoma after therapy: CT and MR imaging," 2003.

[44] S. Woo, C. H. Suh, S. Y. Kim, J. Y. Cho, and S. H. Kim, "Magnetic resonance imaging for detection of parametrial invasion in cervical cancer: an updated systematic review and meta-analysis of the literature between 2012 and 2016," European Radiology, vol. 28, no. 2, pp. 530-541, 2018.

[45] H. Sakurai, Y. Suzuki, T. Nonaka et al., "FDG-PET in the detection of recurrence of uterine cervical carcinoma following radiation therapy-tumor volume and FDG uptake value," Gynecologic Oncology, vol. 100, no. 3, pp. 601-607, 2006.

[46] C. Meads, C. Davenport, S. Małysiak et al., "Evaluating PET$\mathrm{CT}$ in the detection and management of recurrent cervical cancer: systematic reviews of diagnostic accuracy and subjective elicitation," BJOG: An International Journal of $\mathrm{Ob}$ stetrics \& Gynaecology, vol. 121, no. 4, pp. 398-407, 2013. 